\title{
LETTERS
}

doi:10.1017/\$1041610206213474

\section{Diagnosis of Asperger Syndrome in a 66-year-old male presenting with depression}

Asperger Syndrome (AS) is an uncommon condition which is a part of the autistic spectrum disorders group. This condition, first described in 1944 by Hans Asperger was characterized by problems in social integration, verbal communication, empathy and preoccupation with unusual interests (Fitzgerald and Corvin, 2001). Several diagnostic criteria are currently used to diagnose AS, including the ICD-10 (World Health Organization, 1992) and the DSMIV (American Psychiatric Association, 1994), but those proposed by Gillberg are probably closest to Asperger's original description (Fitzgerald and Corvin, 2001). Both the ICD-10 and the DSM require the presence of normal speech and IQ to be present from early life. Gillberg's diagnostic criteria include social impairment, narrow interests, repetitive routines, speech and language peculiarities, non-verbal communication problems and motor clumsiness (Ehlers and Gillberg, 1993).

We report a case of AS that was diagnosed in a 66-year male patient following his referral to old age psychiatry services for depression. He gave written informed consent to the publication of this letter.

A 66-year old Caucasian male was referred to our old age psychiatry department with low mood, irritability, occasional bad temper and withdrawal from the family. On referral he was on Citalopram $60 \mathrm{mg}$ with modest response. As a child he did well at school, but did not mix with others. He had never been good at sport and had never been able to learn to ride a bike. He described himself as bit of a loner and enjoyed his job as a postal worker as this gave an opportunity to enjoy his fascination with numbers. He avoided working in a sorting office because he liked his own company. There was a long history of obsessional traits involving numbers. He was happiest when on his own or listening to his music, and used to collect ties, even though he did not wear them. He expressed difficulties in coping with change and operated within rigid routines. He became upset when his routine was disturbed, for example when his grandchildren were visiting.

His family found that he tended to lash out when out-of-doors, particularly in crowds. On interview he admitted to low mood, reduced appetite, insomnia and poor memory. On mental state examination, there was decreased facial expression with occasional smiles. His conversation was circumstantial, with 
some negative thoughts about the future. There were no deficits elicited on cognitive testing with the Mini-mental State Examination (MMSE) Neuroimaging with Computed Tomography (CT) and Single Photon Emission Computed Tomography (SPECT) revealed no abnormality. A referral to clinical psychology was made to determine whether Cognitive Behavioral Therapy (CBT) could help with his residual symptoms of depression.

During his CBT sessions, it became evident that his rigid and compulsive personality was the key factor in his difficulties. He was found to have an inflexible thinking style and perseverative quality to his speech. He had little regard as to whether the listener was engaged in the conversation and very little eye contact. He scored 31 out 135 on the Friendship questionnaire (Baron-Cohen and Wheelwright, 2003) indicating significant difficulties relating to others and establishing friendships. The Autism Spectrum Quotient (Baron-Cohen and Wheelwright 2001), which assesses social skills, attention switching, and attention to detail, communication and imagination, was also admistered. His score was in the borderline range but scored within the autistic range on the relatives' version of the questionnaire. On the comprehension subtest of the Wescher Adult Intelligence Scale (WAIS-III), he provided literal meaning to the proverbs, indicating rigid thinking. He showed difficulty in object assembly but was in the average range in block design.

The results of psychological testing, positive features from his history and his general presentation supported a diagnosis of AS. As far as we are aware, this is the first report of Asperger Syndrome being diagnosed in an older adult. There are reports of younger adults presenting with other psychiatric presentations (Bankier et al. 1999,) who were found to have AS. AS is still a relatively new syndrome so there may be unrecognized older adults with this condition, particularly as the ICD-10 requires that communicative phrases be used by the age of three or earlier, in order to fulfil the criteria for diagnosis. AS can often be mistaken for obsessive-compulsive disorder or schizoid personality disorder, leading to inappropriate management of these patients. Appropriate structure and support for people with AS can help reduce stress to improve functioning. Family and carers may benefit from formal counselling or group support. One of the difficulties in managing our patient was finding appropriate services to deal with his particular needs. Many of the available day centres for older people cater for people with dementia or functional disorders. Services available for those with AS and their carers in the main cater for children or adolescents. Existing services for older people will need to become better developed in dealing with AS and people with high functioning autism, particularly as in the future the number of older people recognized with these conditions will increase due to greater awareness of these conditions.

It is important to consider Asperger Syndrome in patients who have lifelong difficulties and present with psychiatric symptoms or features of mental illness. 
Recognition of AS can help in managing these patients more appropriately using more behavioural and psychosocial interventions.

\section{References}

American Psychiatric Association (1994). Diagnostic and Statistical Manual of Mental Disorders (4th ed.). Washington, DC: American Psychiatric Association.

Bankier, B., Lenz, G., Gutierrez, L., Gutierrez, K., Bach, M. and Katschnig, H. (1999). A case of Asperger's Syndrome first diagnosed in adulthood. Psychopathology, 32, 43-46.

Baron-Cohen, S., Wheelwright, S., Skinner, R., Martin, J. and Clubley, E. (2001). The autism-spectrum quotient (AQ): evidence from Asperger Syndrome/high functioning autism, males and females, scientists and mathematicians. Fournal of Autism and Developmental Disorders, 31(1), 5-17.

Baron-Cohen, S. and Wheelwright, S. (2003). The friendship questionnaire. An investigation of adults with Asperger Syndrome or high functioning autism, males and females, scientists and mathematicians. Fournal of Autism and Developmental Disorders, 33, 5, 509-517.

Ehlers, S. and Gillberg, C. (1993). The epidemiology of Asperger Syndrome. A total population study. F. Child Psychol Psychiatr, 34, 1327-1350.

Fitzgerald, M. and Corvin, A. (2001). Diagnosis and differential diagnosis of Asperger Syndrome. Advances in Psychiatric Treatment, 7, 310-318.

World Health Organization (1992). International Classification of Diseases (10th ed.) Geneva: WHO.

Anitha Naidu, ${ }^{1}$ Ian James, Elizabeta Mukatoeva-Ladinska AND RUTH BRIEL

${ }^{1}$ Newcastle General Hospital, Castleside Offices, Westgate Road, Newcastle-upon-Tyne NE4 6BE, U.K.

Email: dranithanaidu@gmail.com

\section{Alzheimer's disease in a patient with major depression and excellent performance in concise neuropsychological tests}

During the last decade, the focus of the diagnostics of Alzheimer's disease (AD) has turned to very mild cases. This has been made possible by increasing knowledge of the early symptoms of $\mathrm{AD}$ and the rapid development of diagnostic instruments. Still, the first procedures are clinical assessment and short cognitive tests. Will this be enough or do we still miss cases of AD?

The Mini-mental State Examination (MMSE) is clearly not sensitive enough in diagnosing AD (Nestor and Hodges 2001), but even the CERAD cognitive battery (Welsh et al., 1994) does not always work with very mild cases (Karrasch et al., 2005). More accuracy can be achieved by neuroimaging and biomarkers, but they are not always available and administration of these require a suspicion of a dementing illness. 
We present here a patient with depression and memory complaints in which a concise neuropsychological test did not show any decline in episodic memory, and the diagnosis of $\mathrm{AD}$ was not established until after death. Her husband gave written consent for the publication of this letter.

The patient was a 63-year old woman with hypertension, otherwise healthy and with no history of depression. She had been a teacher of music, and had retired six years earlier because of hip arthritis. Until summer 2003 she had been functioning well, then her mother died and she began to feel unable to do anything. She begun to suffer from insomnia, lost her appetite, began to express unrealistic fears, and her weight decreased $20 \mathrm{~kg}$ in six months. Sertraline was administered for depression and she visited a psychiatrist regularly. She was also examined by a neurologist, without further investigations.

During the autumn her functional capacity declined markedly. She did not prepare any food nor did she take part in any of the household tasks. She was totally hopeless and sometimes also anxious and agitated. Several antidepressants were tried but they did not help.

On January 2004, she came to see another neurologist (KJ). She felt she could neither remember nor do anything, was totally hopeless, expressed wishes to die and was also somewhat restless and bewildered.

At cognitive screening tests her results were normal. The MMSE score was $28 / 30$ (errors in subtraction and figure). Of the three words, she said "I cannot remember any of those words but ", repeating then all the words correctly. On the CERAD neuropsychological test battery, her verbal fluency was normal and naming perfect. At the word-list test of 10 words she had first difficulties in learning them (2-6-8 words in respective rounds), but after a delay she remembered all the 8 words she had learned, and her recognition was $100 \%$. She could also copy and remember all the figures correctly, and the clock-drawing test was normal.

The clinical conclusion was that there were no signs of $\mathrm{AD}$. No brain imaging was thought to be necessary.

One month later, the patient jumped from a sixth floor window and died immediately. A neuropathological autopsy was conducted and clear-cut Alzheimer changes in the brain were found, fairly plentiful neuritic plaques on cortices corresponding to CERAD level C (Mirra et al., 1991) and Braak staging (Braak and Braak, 1991) of the neurofibrillary changes corresponded to level IV.

It is obvious that all patients with subjective memory complaints cannot be examined by modern diagnostic instruments. But it is good to bear in mind that clinical assessment and neuropsychological screening tests do not always reveal very early and atypical cases of $\mathrm{AD}$, especially in people with high education. 
With this patient, all physicians were led astray by the fact that she had a good reason for, and clear clinical symptoms of major depression. She also performed exceptionally well in the CERAD neuropsychological battery. It is evident, though, that she had begun to notice changes in her memory and cognition, and this was manifested in avoiding challenging tasks, deepening her depression and hopelessness.

It is probable that more detailed neuropsychological tests could have revealed cognitive decline, and that neuroimaging or CSF biomarkers would have shown Alzheimer-type changes, but as there was no clear indication, they were not administered.

In her current situation, she did not get any valid explanation for her feelings of devastating memory disorder, and her life came to a sad end. It is of course difficult to know whether the right diagnosis would have made any difference in her life.

This case shows that the diagnosis of early stage of AD can be extremely difficult. Persons with high primary capacity and education can perform well in concise tests. A treatment-resistant major depression with memory complaints and without any history of previous depressive episodes should be considered a potential case of $\mathrm{AD}$.

\section{References}

Braak, H. and Braak, E. (1991). Neuropathological staging of Alzheimer-related changes. Acta Neuropathologica, 82, 239-259.

Karrasch, M., Sinerva, E., Gronholm, P., Rinne, J. and Laine, M. (2005). CERAD test performances in amnestic mild cognitive impairment and Alzheimer's disease. Acta Neurologica Scandinavica, 111, 172-179.

Folstein, M. F., Folstein, S. E. and McHugh, P. R. (1975) "Mini-mental state": a practical method of grading the cognitive state of patients for the clinician. Fournal of Psychiatric Research, 12, 189-198.

Mirra, S. S. et al. (1991). The Consortium to Establish a Registry for Alzheimer's Disease (CERAD). Part II. Standardization of the neuropathological assessment of Alzheimer's disease. Neurology, 41, 479-486.

Nestor, P. and Hodges, J. R. (2001). The clinical approach to assessing patients with early-onset dementia. In Hodges, J. R. (Ed.) Early-Onset Dementia: a Multidisciplinary Approach. Oxford: Oxford University Press.

Welsh, K. A. et al. (1994). The Consortium to Establish a Registry for Alzheimer's Disease (CERAD). Part V. A normative study of the neuropsychological battery. Neurology, 44, $609-614$.

KaTi JUVA $^{1}$ AND Raimo Sulkava ${ }^{2}$

${ }^{1}$ Helsinki University Central Hospital, Division of Psychiatry

Minna Canthin katu 18 A 300250 Helsinki, Finland.

Email: katijuva@katto.kaapeli.fi

${ }^{2}$ University of Kuopio and Rheumatism Foundation Hospital, Heinola, Finland 


\section{Dementia care in Asia - Second Consensus Statement on Quality Services}

As indicated in the Guest Editorial published in this Journal (Chiu and Chiu, 2005), the Consensus Programme on improving the Quality of Life for Asian People with Dementia (QoLDEM) continued its activity in 2005. A second Consensus Statement has been prepared.

This, the second Consensus Statement was prepared at a consensus meeting in Hong Kong in April 29-30th 2005, addressing the domains of service systems, human resources to support quality services, training format, quality care and consumer participation.

We wish to make this Consensus Statement available for promulgation to the field, and it is now published online as supplementary material attached to the electronic version of this letter at www.journals.cambridge.org/jid_IPG.

\section{Reference}

Chiu, H. and Chiu, E. (2005). Dementia Care in Asia. International Psychogeriatrics, 17, 1-2.

EDMONd CHIU* AND Helen CHIU ${ }^{\star \star}$

*Academic Unit for Psychiatry of Old Age, University of Melbourne, Australia.

E-mail: e.chiu@unimelb.edu.au

${ }^{\star \star}$ Department of Psychiatry, The Chinese University of Hong Kong, Hong Kong, SAR,

China. E-mail: helenchiu@cuhk.edu.hk

doi:10.1017/S1041610206243473 First published online 15 Feb 2006

\section{Is culture an independent variable in psychogeriatrics? The case of Japanese and Brazilian elderly}

In a recent article published in the Lancet by the anthropologist Hunt (2005), entitled "Health research: what's culture got to do with it?", we were surprised by the persistent emphasis on possible social explanations for the lower prevalence of depression among Mexican-Americans, while minimizing or even nullifying the potential cultural factors involved.

Due to the social problems that affect Mexican population in the U.S.A., one would expect the prevalence of depression to be higher in this group than among non-Hispanic whites. This is of special interest to psychogeriatrics since, in the globalizing world, cultural factors are supposed to influence the elderly more than the young.

It took time in order for medical researchers to recognize the effects of culture upon a society's health status (Fisher and Fisher, 1961). Take, for instance, 
the extremely low incidence of cysticercosis among Muslim countries, which is attributed solely to the mandatory religious abstinence of pork (Hira et al., 2004). Another obvious example is the past relationship between ritualistic endocannibalism of the brain and "kuru" (a prion disease) in Papua New Guinea (Fisher and Fisher, 1961).

Analogously, but more subtly, there is a large body of evidence that patterns of mental categories and symbols affect the way we perceive and interact with the world (Nisbett et al., 2001). Indeed there are many journals specializing in transcultural psychiatry and psychology.

In a recent research we investigated 421 elderly living in southern Brazil and 411 in Japan (Kyoto), both in rural areas. It was found that in the Brazilian elderly, despite lower mean income and significantly higher prevalences of dependence and comorbidities (data not shown), their Geriatric Depression Scale (GDS) score was lower (2.50) when compared to that of the Japanese (3.35; $\mathrm{P}<.001)$. The gap became more obvious if dependent elders were excluded from the analysis because of the higher dependence in Brazil (vide table S1, published online attached to the electronic version of this letter at www.journals.cambridge.org/jid_IPG). Significant depressive symptomatology (GDS $\geq 6$ ) is observed in $12.6 \%$ of Brazilians and $22.7 \%$ of the Japanese elderly $(\mathrm{P}<.015)$.

This is in accord with Meguro et al. (2001), who found depression to be less common among Japanese-Brazilian elderly living in Brazil than Japanese elderly in Japan.

In Brazil, but not Japan, lower income ( $\leq 50$ percentile) was associated with a $1.74(1.1-2.8, \mathrm{P}: .036)$ times higher odds ratio for significant depressive symptomatology (GDS $\geq 6$ ). This phenomenon is in accord a social inequality much higher in Brazil than Japan.

Quality of Life (QOL) was analyzed through a Visual Analog Scale, varying from 0 (worst) to 100 (best). The average sense of "Life Satisfaction" (LS) was higher among the Brazilian elderly (86.5) than among the Japanese (73.1; $\mathrm{P}<.001)$.

The degree of satisfaction with four domains of QOL (health, family, friends and finances) was also investigated through Visual Analog Scale. A linear regression analysis showed the relative importance of each domain in the LS score. In Brazil, "family" was the single domain which most explained the variability in LS ( $\left.\mathrm{R}^{2}: 27 \%\right)$, followed by "friends," "health" and "finances". Conversely, in Japan "finances" came first $\left(\mathrm{R}^{2}: 26.5 \%\right)$, followed by health, friends and family. For all those variables significance was lower than 0.001 .

In the last year, the World Health Organization (2004) reported a higher prevalence of anxiety and mood disorders in the U.S.A. than in most countries in Latin America, Europe and Asia. 
Besides, in the World Values Survey, which investigated the level of selfperceived happiness/well-being among 79 countries, Mexico was second to Puerto Rico, another Latin-American (LA) country and the U.S.A. ranked fifteenth (Inglehart et al., 2004).

In fact, as a group, Latin American countries scored higher than developed ones. Statistical methods evidenced that, once the economic factor is removed from the model, LA showed to be the most "life satisfaction-driven" cultural area in the world (Inglehart et al., 2004).

In the present study, even though the Brazilian elderly were more dependent, had more their QOL clearly above their Japanese correspondents. Apart from socioeconomic influences, culture was an independent factor associated with higher self-perceived QOL and less depressive symptomatology in Brazil.

Pre-modern anthropology had a long tradition in studying non-European cultures, which were considered less developed (Dunn, 1974). Here, the posture was to confirm how European culture was superior. Like psychotherapists and physicians, anthropologists also should be cautious not to engage unconsciously in (cultural) countertransference (Gorkin, 1986).

An area common to modern anthropology and medicine, Medical Anthropology is a new field of expertise and - together with Cultural Epidemiology - it has been contributing to fill the gap between the human and health sciences, which is one of the paradigms of gerontology.

Culture may be an important independent factor in medicine and especially geriatrics, particularly when psychological domains are in question.

\section{References}

Dunn, S. P. (1974). Prejudice in anthropology: some hidden issues, and suggestions for further research. American Anthropologist, 76, 345-348.

Fisher, A. and Fisher, J. L. (1961). Culture and epidemiology: a theoretical investigation of kuru. Fournal of Health and Human Behavior, 2, 16-25.

Gorkin, M. (1986). Countertransference in cross-cultural psychotherapy: the example of Jewish therapist and Arab patient. Psychiatry, 49, 69-79.

Hira, P. R. et al. (2004). Cysticercosis: imported and autochthonous infections in Kuwait. Transactions of the Royal Society of Tropical Medicine and Hygiene, 98, 233-239.

Hunt, L. M. (2005). Health research: what's culture got to do with it ? Lancet, 336, 617-618.

Inglehart, R., Basanez, M. and Moreno, A. (2004). Human beliefs and values: a cross cultural sourcebook based on the 1999-2002 world values surveys. Mexico City: Michigan.

Meguro, K., Meguro, M. and Caramelli, P. (2001). Environmental chance does not affect dementia prevalence but affects depressive state and physical activity: a transcultural study of Japanese elderly subjects and Japanese elderly immigrants in Brazil. fapanese fournal of Geriatric Psychiatry, 1, 287-295.

Nisbett, E. N. et al. (2001). Culture and systems of thought: holistic versus analytic cognition. Psychological Review, 108, 291-310. 
World Health Organization. (2004). Prevalence, severity and unmet need for treatment of mental disorders in the WHO world mental health survey. Fournal of the American Medical Association, 291, 2581-2590.

Idiane Rosset, ${ }^{1,2}$ Matheus Roriz-Cruz, ${ }^{1,2}$ Teiji SAKagami, ${ }^{3}$ Masayuki Ishine, ${ }^{4}$ Taizo Wada, ${ }^{1}$ Jarbas De SÁ-Roriz, ${ }^{5}$ Rosalina Partezani-Rodrigues, ${ }^{5}$ Antonio C. De Souza, ${ }^{6}$ Kiyohito Okumiya, ${ }^{4}$ TORU Kita ${ }^{1}$ and Kozo Matsubayashi Md. ${ }^{4}$

Kyoto University, Japan: ${ }^{1}$ Department of Geriatrics. ${ }^{2}$ Department of Neurology and Research Institute for Neuroscience of Aging. ${ }^{3}$ Department of Psychiatry. Divisions of Geriatric Psychiatry and Transcultural Psychiatry. ${ }^{4}$ Department of Field Medicine and Research Institute of Humanity and Nature. ${ }^{5}$ Departamentos de Geriatria e Enfermagem Gerontológica. Universidade de São Paulo - RB, Brazil. ${ }^{6}$ Instituto de Geriatria. Universidade Católica do Rio Grande do Sul, Brazil.

\section{Observations from a 14-week open-label trial with memantine suggest variable response on behavioral symptoms and cognition, depending on former treatment of AD}

Memantine, an uncompetitive $\mathrm{N}$-methyl D-aspartate (NMDA) receptor antagonist, is currently the only treatment licenced for moderately severe to severe Alzheimer's Disease (AD). Memantine is effective on cognitive symptoms (Reisberg et al., 2003; Tariot et al., 2004; Winblad and Portis, 1999), and in a post-hoc analysis of behavioral symptoms, memantine showed beneficial effects particularly on agitation/aggression (Gauthier et al., 2005).

For mild to moderately severe AD, cholinesterase inhibitors (AChEIs) are licenced. Patients, who have been treated with AChEIs during the early stage of the disease and show progression of $\mathrm{AD}$ under treatment, can be offered a switch from an AChEI to memantine. However, there are no data available on the effect of memantine in relation to former treatment of AD.

We present a prospective 14-weeks multi-centre open-label study in the Netherlands of the efficacy and tolerability of memantine treatment in DSM-IVdefined $\mathrm{AD}$ outpatients, in relation to former treatment of $\mathrm{AD}$. Memantine was prescribed in a standard titration, starting at $5 \mathrm{mg} /$ day, and increasing $5 \mathrm{mg} /$ day every week until $20 \mathrm{mg} /$ day from week 4 until the end of the study. After inclusion in the study, the patient visited the hospital in weeks 2,8 and 14. Primary efficacy variables were mean change from baseline to week 14 on Mini-mental State Examination (MMSE), Neuropsychiatric Inventory (NPI) including carer distress, and a Visual Analogue Scale (VAS) for well-being, evaluated by caregiver. Besides the NPI, clusters of BPSD symptoms are asked 
for (see Table S1, published online as supplementary material attached to the electronic version of this letter at www.journals.cambridge.org/jid_IPG).

Statistical analysis was performed using the SPSS version 11.5 software. In this analysis, all available data of patients are included by visit (Observed Cases, OC). Changes from baseline in mean MMSE and NPI scores at week 14 (OC) were compared using the paired $t$-test. Statistical significance was accepted for $\mathrm{p}<0.05$.

Forty-seven patients were included, 63\% women, mean age 76.3 years old (SD 7.4), 60\% with relevant co-morbidity, mean Global Deterioration Scale score 5.4 (SD 0.76). Patients were divided into two groups: group $\mathrm{B}(\mathrm{N}=18)$, unsuccessfully treated with cholinesterase inhibitor (AChEI) prior to this study, and group $\mathrm{N}(\mathrm{N}=29)$, which had not been treated before.

Former treatment of AChEI was stopped due to lack of efficacy $(50 \%)$, adverse events (50\%) and other reasons (16\%).

Forty-one patients completed the trial. Mean MMSE increased nonsignificantly by 0.4 points at the end of the study (OC, Figure S1). NPI severity decreased significantly after 2,8 and 14 weeks $(\mathrm{p}<0.05)$ (Figure S2), presence of BPSD symptoms decreased significantly at weeks 8 and $14(\mathrm{p}<0.05)$ and the VAS score showed no difference $(p=0.392)$. Figures $S 1$ and S2 published online as supplementary material attached to the electronic version of this letter at www.journals.cambridge.org/jid_IPG.

Mean baseline MMSE, NPI severity and carer distress, and VAS for group N $(\mathrm{N}=29)$ were respectively $11.7 ; 9.6$ and $9.3 ; 52 \mathrm{~mm}$ and for group $\mathrm{B}(\mathrm{N}=18$ prematurely stopped patients included) respectively $11.8 ; 6.8$ and $4.9 ; 52 \mathrm{~mm}$.

After 14 weeks mean MMSE increased 1.1 points $(p=0.069)$ in group $\mathrm{N}$, and decreased in group B 1.2 points $(p=0.212)$. The NPI decreased 2.4 $(p=0.049)$ in group $\mathrm{N}$, and $3.9(p=0.034)$ in group $\mathrm{B}$, especially the nonpsychotic symptoms aggression/agitation and depression/anxiety. The total use of antipsychotics remained stable during the study $(17.1 \%$ at baseline and $19.5 \%$ at week 14). The VAS scores did not show any difference between the groups.

Memantine was well tolerated during the study period (Table S2, published online attached to the electronic version of this letter at www.journals.cambridge.org/jid_IPG). During the trial period, mean weight non-significantly increased from $73.9 \mathrm{~kg}$ to $74.6 \mathrm{~kg}$. Not related to the use of memantine, 3 patients stopped with the study drug. Three more patients dropped out of the study due to apathy, dizziness and somnolence. All these patients were previously treated with CI (Group B, 17\%) and the reasons for stopping former CI treatment were both adverse events and lack of efficacy.

In conclusion, the well-tolerated memantine treatment resulted in improvement on behavioral disturbances measured by the NPI. However, our data suggest variable response on behavioral symptoms and cognition, depending 
on former treatment of $\mathrm{AD}$. Further studies are required to confirm the effect of memantine in relation to former AD treatment.

\section{Acknowledgement}

This letter was written on behalf of the investigators of Memantine Observation Study Netherlands.

\section{References}

Gauthier, S., Wirth, Y. and Möbius, H. J. (2005). Effects of memantine on behavioural symptoms in Alzheimer's disease patients: an analysis of the Neuropsychiatric Inventory (NPI) data of two randomised, controlled studies. International fournal of Geriatric Psychiatry, 220, 459-464.

Reisberg, B., Doody, R., Stöffler, A., Schmitt, F., Ferris, S. and Möbius, H. J. (2003). Memantine in moderate-to-severe Alzheimer's disease. New England fournal of Medicine, 348, 1333-1341.

Tariot, P. N., Farlow, M. R., Grossberg, G. T., Graham, S. M., McDonald, S. and Gergel, I. (2004). Memantine treatment in patients with moderate to severe Alzheimer disease already receiving donepezil. A randomised controlled trial. fournal of the American Medical Association, 291, 317-24.

Winblad, B. and Portis, N. (1999). Memantine in severe dementia: results of the M-BEST study (benefit and efficacy in severely demented patients during treatment with memantine). International Fournal of Geriatric Psychiatry, 14, 135-146.

Paul L. J. Dautzenberg, ${ }^{1}$ Cees J. Wouters

AND JANET E. M. BOOTSMA

Department of Geriatrics, Jeroen Bosch Hospital, GV's-Hertogenbosch, The Netherlands.

${ }^{1}$ E-mail: p.dautzenberg@jbz.nl

\section{Language-relevant interventions for verbally disruptive behaviors in dementia}

Verbally disruptive behaviors (VDBs) are relatively common in persons with dementia in residential care and are of concern, as they may indicate distress in the older person and disturb those in close vicinity (Cohen-Mansfield and Werner, 1997). While the causes of VDB are complex, one factor that has been identified is social deprivation, with the behavior indicating an unmet need that is unable to be expressed (Cohen-Mansfield and Werner, 1997). This may be particularly relevant to persons from linguistically diverse backgrounds, who 
commonly revert to their original language as their dementia progresses (Lewis and Kirchener, 1996). Consequently, persons with dementia and low levels of English language proficiency residing in mainstream care are likely to be involved in limited social interaction and have difficulty communicating their needs.

Our past research compared the effect of social interaction and listening to music in Italian or English language on the VDBs of an Italian woman with dementia (Runci et al., 1999). The intervention was more effective, in terms of decreasing disruptive verbalizations and increasing meaningful communication, when it was in Italian. While findings offered some initial evidence for the importance of providing language-relevant interventions, the agent of change was not clear and only a single participant was involved. The aim in the current project was to extend our previous research by providing a more specific intervention in a series of single-case designs. Consent was obtained for the publication of these case details.

Participants were three older Italian-background persons with dementia, who were residing in Melbourne aged-care facilities and were considered to be "verbally disruptive" by staff at their facility. The first participant ("Mr. A") was a 70 -year-old male diagnosed with vascular dementia. He received a score of $12 / 30$ on the Italian language version of the Mini-mental State Examination (MMSE; Turrina et al., 1993). “Ms. B.” was a 79-year-old female with a probable diagnosis of Alzheimer's disease. She was unable to respond sensibly to any of the questions on the MMSE and received a score of zero. "Mr. C." was an 80-year-old male with a probable diagnosis of Alzheimer's disease. He also scored zero on the MMSE. All participants were prescribed psychotropic medication, which were unaltered during the study.

Participants were observed three times per day for 20-minute periods, 2 days per week, for a total period of 8 weeks ( 4 weeks baseline and 4 weeks intervention). During the baseline, the researcher sat in the day room and recorded verbalizations every 30 seconds using a one-zero time-sampling procedure. Verbalization categories were "non-disruptive verbalizations" (nonrepetitive verbalizations at normal pitch in English, Italian or "language unclear", where language could not be deciphered as English or Italian), "no verbalizations" or "VDB". Mr. A.'s VDBs included “directed aggressive verbalizations" (for example, swearing at staff or co-residents), repetitive requests and "moaning". Ms. B.'s VDBs included “directed aggressive verbalizations" and "repetitive requests". Mr. C.'s VDB was "wailing” (a prolonged, inarticulate, high pitched crying sound).

During the intervention phase, the researcher spent the 20-minute periods sitting and speaking to each participant and the interaction was conducted in either Italian or English. Languages were randomly ordered to days, with four days of interaction in each language. 
A small cassette recorder was used to tape verbalizations, with a microphone attached to the researcher. Six raters listened to tapes for randomly selected segments of recordings.

$\mathrm{An} A B$ design was used, with randomly ordered alternating treatments in the intervention phase. A randomization test (performed using a statistical package for single-case designs) was employed to compare verbalizations during the English and Italian interventions.

Inter-observer reliability kappa co-efficients for verbalization categories ranged from 0.94 to 0.98 .

Mr. A. exhibited a non-significant decrease in VDB during the Italian intervention $(\mathrm{B}-\mathrm{A}=9.25, p>0.05)$, however the $p$-value was approaching significance $(p=0.057)$ (for more detail see Table S1, published online as supplementary material attached to the electronic version of this letter at the journal website www.journals.cambridge.org/jid_IPG). Conversely, a significantly higher level of non-disruptive verbalizations (directed talking in Italian) was observed in the Italian intervention $(\mathrm{A}-\mathrm{B}=25.25, p<0.05)$.

There was a very low level of VDB during both interventions for Ms. B. $(\mathrm{A}-\mathrm{B}=1.25, p>0.05)$. She exhibited a significant increase of non-disruptive verbalizations (directed talking in Italian and "language unclear" verbalizations) in the Italian intervention $(\mathrm{A}-\mathrm{B}=22.25, p<.05)$.

Mr. C.'s results showed a non-significant reduction of $\operatorname{VDB}(\mathrm{B}-\mathrm{A}=6.5$, $p>0.05$ ) and a significantly higher level of non-disruptive verbalizations ("language unclear") when the intervention was in Italian $(\mathrm{A}-\mathrm{B}=10.5, p<0.05)$.

Findings demonstrated a significant increase in non-disruptive behaviors and no change in VDB for the language-relevant (Italian) intervention. However, Mr. A.'s VDB results did show a trend towards an improved effect of the Italian intervention.

Our study relied on quantitative measures and statistical significance as indicators of treatment efficacy. It would be useful to include measures of clinical significance to demonstrate the benefit of social interaction in the daily lives of participants. It would also be beneficial to incorporate information from others who are regularly in the vicinity of the person with dementia to provide a greater understanding of the impact of a change in verbalizations.

Our project included three participants with differing levels of cognitive functioning. It appears that the usefulness of verbal interaction for persons with severe dementia who exhibit VDBs may be limited. It may be that other types of psychosocial interventions that do not rely on language ability may be more effective for this population.

The multifactorial aetiology of VDBs and the need for a comprehensive intervention has been highlighted previously (Meares and Draper, 1999). We used the same intervention for all participants, to focus on the participants' 
original language and enable replication of findings. The underlying assumption was that the lack of language-relevant social stimulation may have been a contributing factor to VDBs, and this may not have been the case.

It is important to highlight, however, that our findings did provide support for the use of language-relevant interaction, in terms of the increase in nondisruptive verbalizations. Even Mr. C., the most impaired participant, exhibited a significantly higher level of non-disruptive verbalizations during the Italian interaction. It may be that our dependent measure underestimated the value of the intervention, due to the compromised verbal abilities of our participants.

Our aim was to present some initial data to provide impetus for further, larger scale research. Future research could incorporate measures of clinical significance, include a comprehensive assessment of VDBs, and design interventions accordingly.

\section{References}

Cohen-Mansfield, J. and Werner, P. (1997). Management of verbally disruptive behaviours in nursing home residents. Fournals of Gerontology, 52A, M369-M377.

Lewis, I. D. and Kirchener, S. (1996). Dealing with ethnic diversity in nursing homes. In G. Yeo and D. Gallagher-Thompson (Eds.), Ethnicity and the Dementias. Washington, DC: Taylor and Francis.

Meares, S. and Draper, B. (1999). Treatment of vocally disruptive behaviour of multifactorial aetiology. International fournal of Geriatric Psychiatry, 14, 285-290.

Runci, S., Doyle, C. and Redman, J. (1999). An empirical test of language-relevant interventions for dementia. International Psychogeriatrics, 11, 301-311.

Turrina, C., Dewey, M., Siani, R., Saviotti, F., Marchione, N. and Siciliani, O. (1993). Performance of the Italian version of the Mini-mental State Examination in day hospital geriatric medical patients. International Fournal of Geriatric Psychiatry, 8, 649-654.

SusAnNAH J. RUnCI, JENNIFER R. REDMAN ${ }^{2}$ AND

DANIEL W. O'CONNOR ${ }^{1,3}$

${ }^{1}$ Aged Mental Health Research Group, Department of Psychological Medicine, Monash University, Kingston Centre, Warrigal Rd, Cheltenham, VIC 3192, Australia.

Email: Susannah.Runci@med.monash.edu.au

${ }^{2}$ Associate Professor, Department of Psychology, Monash University, Australia.

${ }^{3}$ Professor of Psychological Medicine.

doi:10.1017/S1041610206273472 First published online 18 Jan 2006

\section{Disability and post-traumatic psychopathology in Turkish elderly after a major earthquake}

An earthquake with a magnitude of 7.4 on the Richter scale left devastating effects in the Marmara region of Turkey in August 1999 (Laor et al., 2002). 
Reported global clinical experience and scientific data to guide interventions for mental health needs of the elderly after a mass disaster are limited. As a response to trauma, presumably older adults may be more likely to re-experience the traumatizing event and exhibit emotional blunting, avoidance-behavior and exaggerated physiological arousal. Besides these symptoms, decrease in functioning and worsening of medical illnesses may be seen after major trauma in the elderly (Weintraub and Ruskin, 2003). In previous reports, data were insufficient for psychiatric diagnosis post-earthquake and disability associated with trauma. We hypothesized that elderly people who experienced the earthquake at the epicenter would be psychologically affected more significantly than elderly people living at least $150 \mathrm{~km}$. away from the epicenter. This effect would be reflected as higher Post-traumatic stress disorder (PTSD) scores, more PTSD, or PTSD comorbid with depression diagnoses and higher disability levels in the affected population.

Twenty-five older adults (mean age $=68.4 \pm 5.07$ ) from Adapazari who were relocated at a pre-fabricated village (epicenter group) and 22 elderly (mean age $=76.52 \pm 5.17$ ) who live in an Istanbul nursing home (non-epicenter group) and experienced the earthquake at much less intensity, were included in our study. Those with dementia and with significant visual or hearing impairment were excluded from the study. Nine months after the earthquake, each patient was administered a series of scales: Davidson Trauma Scale (DTS), Brief Post-traumatic Grief Scale (BPGS) and Brief Post-traumatic Dissociation Scale (BPDS), WHO Disability Assessment Scale (WHO-DAS-II) and Mini-mental State Examination (MMSE). DTS is a self-report rating scale, which consists of 17 items corresponding to DSM-IV criteria of PTSD, yielding B, C and D subscores (Davidson et al., 1999). BPGS and BPDS were developed by a part of our group working with adults and children at the same site in Adapazari (Laor et al., 2002). WHO-DAS - II is a disability-assessment scale whose reliability and validity in Turkish has been established (Ulug et al., 2001). Upon completion of the scales, a geriatric psychiatrist, who was blind to scales, performed semistructured clinical diagnostic interviews with each subject, enquiring about major DSM-IV diagnostic categories. Differences on the scales between epicenter and non-epicenter groups, and among different diagnostic groups in the epicenter group were compared using ANOVA. Comparison of diagnostic frequency between two groups were done with Fisher's Exact Test $(P<0.05$ accepted for significance). Bonferroni correction was applied to all statistical results, because of the small number of subjects and the multiple comparisons. $(P<0.007$ was set for significance). Pearson's correlational analysis was done between all used scales.

Demographic data and scale scores are given in more detail in Tables S1-S3 published as supplementary material attached to the electronic version of this letter online at www.journals.cambridge.org/jid_IPG. 
Total DTS, DTSb, DTSc, DTSd, BPGS and BPDS scores were significantly higher $(p<0.005)$ in the epicenter group compared to the non-epicenter group. In the epicenter group, PTSD alone (28\%) and PTSD comorbid with major depression $(32 \%)$ diagnoses were significantly more prevalent $(p=0.01$ and $p=0.004$, respectively). A comparison between the individuals with major depression in the epicenter and the control group showed higher levels of trauma-related symptoms on DTS $(p<0.005)$, higher levels of grief on BPGS $(p<0.005)$ and higher levels of disability on DAS-II $(p=0.005)$ in the epicenter group.

In post-hoc analyses, the individuals within the epicenter group who received a diagnosis of PTSD had significantly $(p<0.007)$ higher scores on DTS, DTSc, DASII, BPGS than the individuals having no diagnosis. The second comparison showed that individuals with major depression had significantly $(p<0.005)$ higher scores on DTSc and DAS-II. A post-hoc correlational analysis performed in the epicenter group showed significant and positive intercorrelations on, BPGS, DAS-II, DTS and DTSc scales (Pearson's $\mathrm{r}=0.591 .-0.874$, $p<.007)$.

This study shows that older adults who experienced the earthquake at the epicenter (in contrast to a group of nursing-home residents far from the epicenter) had higher scores on symptom scales measuring psychological trauma, trauma-related grief and dissociation. The epicenter group, as hypothesized, displayed a higher prevalence of DSM-IV diagnoses of PTSD and PTSD comorbid with depression. The results parallel previous research in showing the association between the proximity to the epicenter and the severity of traumarelated symptoms. Avoidance and numbness behaviors and grief were most prevalent among the subjects with PTSD in contrast to the ones without such diagnosis. However, after the earthquake in Armenia, it was reported that elderly survivors displayed higher arousal symptoms and lower intrusive symptoms than younger persons (Goenjian et al. 1994). Avoidance and numbness behaviors may be restrict the quality of life in individuals who experience them, especially by limiting interpersonal contact and mobility, with adverse consequences in social life. We observed that most individuals in the disaster area may either talk about the effects of the earthquake in a repetitive perseverative fashion, or totally avoid talking about what happened to them and their loved ones, both ways being ineffective in coping with the trauma. Disability is an important concept in understanding the relationship between psychopathology and functioning. Being at the epicenter per se, in contrast to our expectations, was not sufficient to produce a significant disability. Our post-hoc analyses, however, indicated that individuals from the epicenter group with diagnoses of major depression had higher levels of disability than the non-epicenter group with depression. These findings suggest that being at the epicenter has an additive effect on 
disability, at least in the presence of depression. The increased disability among the individuals from the epicenter, diagnosed with both depression and PTSD, in contrast to individuals from the epicenter without any diagnoses, provides an indirect support to the psychopathology and disability-generating effect of being at the epicenter. These conclusions, however, remain speculative due to the small sample size, limiting more definitive analyses. In conclusion, our results support the hypothesis that elderly people in an earthquake area are vulnerable to psychiatric illnesses and may develop disability due to depression or PTSD. Special prevention and intervention programs for elderly who were exposed directly to earthquakes should be developed.

\section{References}

Davidson, J. R. et al. (1997). Assessment of a new self-rating scale for post-traumatic stress disorder. Psychological Medicine, 27, 153-160.

Goenjian, K. G. et al. (1994). Post-traumatic stress disorder in elderly and younger adults after the earthquake. American fournal of Psychiatry, 151, 895-901.

Laor, N., Wolmer, L., Kora, M., Yucel, D., Spirman, S. and Yazgan, Y. (2002). Post-traumatic, dissociative and grief symptoms in Turkish children exposed to the 1999 earthquakes. Fournal of Nervous and Mental Disorders, 190(12), 824-832.

Ulug, B., Ertugrul, A., Gogus, A. and Kabakci, E. (2001). Reliability and Validity of the Turkish Version of the World Health Organisation Disability Assessment Schedule-II (WHO-DAS-II) in Schizophrenia. Turk Psikiyatri Derg, 12, 121-130.

Weintraub, D., and Ruskin, P. E. (1999). Post-traumatic stress disorder in the elderly: a review. Harvard Review of Psychiatry, 7, 144-152.

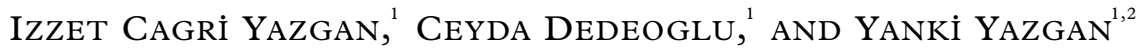

${ }^{1}$ Marmara University Medical Faculty, Department of Psychiatry, Altunizade, Istanbul, Turkey. Email: cgyazgan@yahoo.com

${ }^{2}$ Yale Child Study Center, New Haven, CT, U.S.A.

doi:10.1017/\$1041610206283479

\section{Quetiapine for delusional jealousy in a deaf elderly patient}

Psychotic symptoms are occasionally associated with deafness (Harry and Favazza, 1984). We report a case in which the delusional jealousy of a deaf elderly man was successfully managed with quetiapine without adverse effects. The patient gave written informed consent to publication of the details of his case.

A 62-year-old man visited our outpatient clinic in April, 2005. He had not been able to hear and speak since the age of 4 . One month before visiting our clinic, he became irritable abruptly. He claimed that his wife had been unfaithful by being intimate with one of his relatives. He conversed this to his family through 
gestures and in writing. Subsequently, he found it difficult to sleep and started to act violently toward his wife.

On MRI and a medical work-up, no brain lesions or medical problems could be detected. His Brief Psychiatric Rating Scale (BPRS) score was 62. He was diagnosed with DSM-IV delusional disorder and treatment was started with quetiapine, $50 \mathrm{mg}$, and zolpidem, $10 \mathrm{mg}$, at bedtime. On the third day after treatment, his symptoms decreased remarkably: he did not express any delusional thoughts and his regular sleeping pattern resumed.

Nine days after treatment, the BPRS score was 47 , and three months after treatment, his BPRS score was 37 . He did not develop any severe adverse effects, such as extrapyramidal symptoms.

An association between deafness and delusion in old age has been repeatedly confirmed (Lebuffe and Lebuffe, 1979; Fuchs, 1993). However, the number of studies regarding treatment of elderly, deaf psychotic patients is few, responses to conventional antipsychotic agents have been variable and extrapyramidal and other side-effects have limited their use (David and Fernandez, 2000).

One study showed that quetiapine was useful for the treatment of hypnogogic musical release hallucinations in a deaf elderly person (David and Fernandez, 2000). Our case shows that low-dose quetiapine is also effective in treating delusional jealousy in a deaf elderly patient without adverse effects. We recommend further well-controlled studies regarding the treatment of psychosis associated with sensory impairments.

\section{References}

David, R. R. and Fernandez, H. H. (2000). Quetiapine for hypnogogic musical release hallucinations. Fournal of Geriatric Psychiatry and Neurology, 13, 210-211.

Fuchs, T. (1993). Delusion syndromes in sensory impediment-overview and model presentation. Fortschrift Neurologie und Psychiatrie, 61, 257-266.

Harry, B. and Favazza, A. R. (1984). Brief reactive psychosis in a deaf man. American fournal of Psychiatry, 141, 898-899.

Lebuffe, F. P. and Lebuffe, L. A. (1979). Psychiatric aspects of deafness. Primary Care, 6, $295-310$.

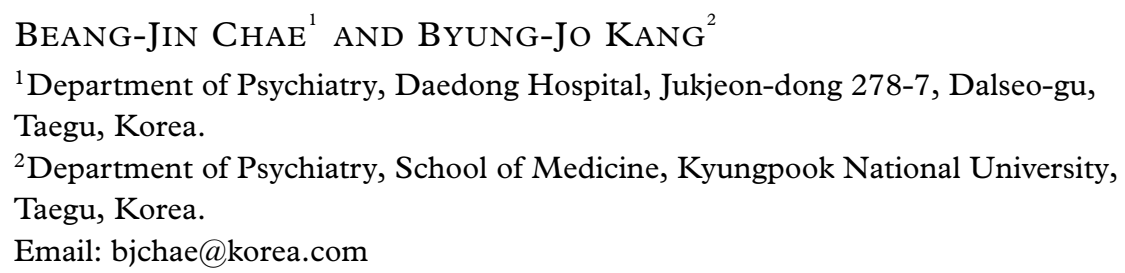

